\title{
Experiencia de creación de una Comunidad Educativa Virtual
}

\author{
(Experience about creation of Educational Virtual Community)
}

ANTONIO Viedma RoJas*

(UNED, España)

RESUMEN: En este artículo se analiza la experiencia de una práctica docente llevada a cabo por un equipo formado por profesores y alumnos de la asignatura Técnicas de Investigación Social en la Facultad de Ciencias Politicas y Sociología de la Universidad Nacional de Educación a Distancia (UNED) en España. El uso de Internet ha permitido ofrecer a los alumnos la posibilidad de realizar una investigación social real. Esta actividad forma parte de una estrategia didáctica más amplia. Con ella, y sin que sea éste un objetivo exhaustivo, hemos pretendido abordar uno de los aspectos menos frecuentes en la práctica habitual de la enseñanza a distancia: la realización de prácticas reales en equipo.

En principio, muchos de los objetivos propuestos se han cumplido. Sin embargo, esto no impide concluir que al igual que en otros campos, Internet se ha mostrado más como un complemento que como un canal de relación exclusivo. En definitiva, podríamos decir que virtualidad y realidad deben unirse para conseguir mejorar la calidad de la enseñanza a distancia.

Enseñanza a distancia a través de Internet - Didáctica de investigación social - Prácticas de investigación social - Comunidad educativa virtual

ABSTRACT: In this article the experience of teaching practice is analysed carried out by a team formed by professors and students of the subject: Technical of Social Research in the Faculty of Political Sciences and Sociology of the Universidad Nacional de Educación a Distancia (UNED) Spain. The use of Internet has allowed to offer the students the possibility to carry out a real research. This activity is part of a wider didactic strategy. With her, and without it is this an exhaustive objective, we have sought to approach one of the aspects less usual in the habitual practices from distance teaching: doing real practices by team.

In principle, we believe that many of the proposed objectives has been completed. However this it doesn't impede to conclude that, the same as in other fields, Internet has been shown more as a complement that as an exclusive relationship channel. To sum up we could say that, virtuality and reality may unite to be able to improve the quality from the teaching to distance.

Teaching to Distance through Internet - Didactics of Social Research-Practical of Social Research - Educational Virtual Community

* La experiencia que se presenta ha sido llevada a cabo por los profesores: Pío Navarro, Javier Callejo, Jesús Gutiérrez y Antonio Viedma. 


\section{INTRODUCCIÓN}

En este artículo se analiza la experiencia de una práctica docente llevada a cabo por un equipo formado por profesores y alumnos de la asignatura Técnicas de Investigación Social en la Facultad de Ciencias Políticas y Sociología de la Universidad Nacional de Educación a Distancia (UNED) en España. En el contexto de la asignatura, este trabajo de investigación supone una apuesta por el desarrollo y la aplicación de materiales didácticos diseñados ad-hoc, para ser utilizados a través de Internet. El uso de este medio ha permitido ofrecer a los alumnos la posibilidad de realizar una investigación real. Esta actividad forma parte de una estrategia didáctica más amplia. Con ella, y sin que sea éste un objetivo exhaustivo, hemos pretendido abordar uno de los aspectos menos frecuentes en la práctica de la enseñanza a distancia: la realización de prácticas reales en equipo.

El modelo de enseñanza que propone la UNED se basa en una estructura de apoyo docente mixto. Los alumnos tienen dos alternativas para recibir ayuda y orientación. La primera, haciendo uso de los «Centros Asociados» en los que existe para tal efecto la figura del "profesor tutor»; la segunda, a través del contacto directo con los profesores de la asignatura en la «Sede Central». No obstante, y aunque muchos de los alumnos utilizan estos centros o los contactos con los profesores con cierta asiduidad, la importancia de otros materiales de orientación como las «Guías Didácticas», las «Adendas», y otros textos publicados por la propia UNED, suponen para el alumno una referencia clave a la hora de realizar sus estudios. (Callejo 2000)

En síntesis, esta fórmula proporciona, por un lado, referentes reales de la universidad en su entorno físico y social, aunque a veces el centro asociado sea sólo el punto de reunión para realizar los exámenes o conocer a compañeros. Por otro lado, referentes de consulta y evaluación a distancia de sus conocimientos en relación con el profesorado de la denominada «Sede Central». Parece lógico pensar que es, en el desarrollo de este segundo apartado menos inmediato y por lo tanto, más «virtual»', en el que nos corresponde a los docentes responsables de la asignatura aplicar todos nuestros esfuerzos.

La propuesta realizada por el equipo docente se englobaba dentro de la actividad normal de la asignatura. La importancia de la realización de prácticas, se justifica plenamente en una materia cuyos contenidos teóricos cobran más sentido cuando se desciende al diseño de la investigación y a las prácticas del trabajo de campo. Esta relevancia de la práctica, se valora en la asignatura con un $20 \%$ de la nota final: un 10\% para un trabajo en el que se ha de diseñar un proyecto de investigación o un cuestionario (según sea la primera o segunda presencial) y,

\footnotetext{
${ }^{1}$ Entendiendo por virtual lo que propone Lévy (1999) «lo virtual no se opone a lo real sino a lo ac-
} tual: virtualidad y actualidad sólo son dos maneras de ser diferentes». 
el otro 10\%, con la participación en la investigación a la que nos referiremos en este artículo. En definitiva, dos cuestiones a tener en cuenta con respecto a la participación: la primera, que la intervención en la investigación es voluntaria; la segunda, que el valor que supone en la nota final para el alumno ${ }^{2}$ no puede justificar exclusivamente su colaboración en la misma.

A efectos clasificatorios dividiremos la exposición de este trabajo en cuatro partes: en la primera, explicaremos los objetivos de la propuesta; en la segunda, realizaremos una descripción de la experiencia, de los materiales creados y del si$t e^{3}$ construido, en la tercera, expondremos una síntesis de los resultado obtenidos. Por último, concluiremos con una reflexión orientada más hacia lo social que a lo técnico. Esta revisión abundará en los errores cometidos. Desde este punto de vista crítico, tendremos la oportunidad de ofrecer algunas propuestas que ayuden al desarrollo futuro de nuevas experiencias que utilicen Internet, como medio de aplicación de materiales didácticos a distancia.

\section{OBJETIVOS DE LA EXPERIENCIA}

Más allá de la realización práctica de la investigación o de mostrar el espacio como una base de información relevante para la asignatura, nos planteamos que el objetivo docente fundamental de la experiencia sería la creación de una «comunidad educativa virtual» (CEV).

El reconocimiento del profesor y de los compañeros como actores partícipes de una misma experiencia, la intervención en la construcción de la materia de estudio y la incorporación flexible de tareas y materiales docentes, nos alejan de los sistemas educativos a distancia de corte taylorista. En síntesis, podríamos definir la CEV como una comunidad formada por alumnos y profesores que utilizan para relacionarse un espacio de comunicación e interacción electrónico en el que, a través de diferentes formas sincrónicas y asincrónicas de comunicación individual y grupal, comparten experiencias docentes que incorporan flexibilidad a la metodología de la enseñanza a distancia. La construcción de la CEV se alimenta de las relaciones dialécticas que se producen en el seno de la misma. Lógicamente, el éxito o el fracaso depende de las aportaciones conjuntas de los participantes.

\footnotetext{
${ }^{2}$ Aunque está claro que la no participación en alguno de los trabajos prácticos excluye la posibilidad de obtener la máxima nota. Debemos aclarar también que esta conclusión ha sido extraída de los propios comentarios que los alumnos nos han realizado al respecto. Para poder entender los motivos que han llevado a éstos a participar, se puede realizar una lectura de sus presentaciones en foro de la asignatura en la URL: http:/ www.uned.es/112013.

${ }^{3}$ Tal como se usa habitualmente en los círculos especializados y, por cuestiones de economía del lenguaje, utilizaremos el concepto «site» para hablar del espacio total creado en la «web» para soportar la experiencia.
} 
Es importante señalar también que existen diferencias muy claras con las «comunidades virtuales» de otros espacios electrónicos (Rheingold 1994). A diferencia de éstas, en las que la no existe autoría de la comunicación, el acceso a la participación sólo está delimitado por las características públicas o privadas del espacio electrónico en el que se desarrollan y, los grupos permanecen siempre abiertos a nuevas incorporaciones; las CEV se forman sobre la identidad de sus miembros, la participación limitada a éstos y la construcción de grupos cerrados de trabajo.

No obstante, y aunque el objetivo fundamental era el expresado anteriormente, detallaremos a continuación los objetivos particulares concretos en función del ámbito en el que éstos se apliquen.

\subsection{EN EL ÁMBITO DE LA DOCENCIA}

D Aunar de manera didáctica los contenidos teóricos de la asignatura con la práctica de una investigación real.

D Dirigir y supervisar directamente dicha práctica por los profesores de la asignatura.

- Contribuir a incrementar y renovar sistemáticamente la parte práctica de la asignatura (en cada curso se hará un propuesta de tema a investigar con una metodología diferente).

D Incorporar nuevos contenidos teóricos a la asignatura.

\subsection{EN EL ÁMBITO DE LA INVESTIGACIÓN}

Dromover un espacio propio para la investigación social con fines universitarios.

D Obtener datos empíricos de valor para uso y consulta interna de alumnos y profesores.

D Contribuir al proceso formativo de futuros investigadores.

D Complementar y estimular las labores de investigación del Departamento

\subsection{EN EL ÁMBITO DE LA COMUNICACIÓN Y LA PARTICIPACIÓN}

Conseguir que el alumno exprese públicamente sus dudas y opiniones al equipo docente.

D Establecer la idea de una comunidad o grupo de investigación entre los alumnos (equipo investigador). 
D Promover la integración del alumno en un grupo de trabajo y estudio.

- Aproximar la figura del profesor al alumno, manteniendo un conocimiento y contacto totalmente directo.

Domentar y estimular la implicación del alumno en la asignatura.

- Crear un núcleo o tema de discusión permanente entre alumnos y profesores de la asignatura.

\section{DESCRIPCION DE LA EXPERIENCIA}

\subsection{COMUNICACIÓN DE LA PROPUESTA A LOS ALUMNOS}

En septiembre del año 2000, y ante la difusión masiva del acceso a Internet, consideramos la posibilidad de realizar prácticas de investigación con los alumnos utilizando esta vía. Durante varios meses fuimos realizando un esbozo del diseño y la estructura del site, preparamos algunos materiales y reflexionamos acerca de la forma en que la experiencia sería dirigida. En definitiva, en esta etapa se organizaron los aspectos comunicativos, interactivos y de seguridad del site.

Entre los meses de febrero y marzo se cierra cada año en la UNED la «Guía de la asignatura» para el próximo curso escolar. En esta «Guía» se incluyen todos los contenidos de la asignatura, los trabajos a realizar, la formas de calificar los exámenes y las orientaciones didácticas más importantes. Esta forma de incorporar modificaciones garantiza la comunicación a todo el alumnado. Esto significa, que todo lo publicado en la «Guía» rige la actividad escolar para todo el curso. Para nosotros, éste suponía el primer plazo a cubrir.

En aquel momento, no teníamos todos los materiales disponibles, pero publicamos la propuesta sabiendo que, en el peor de los casos, podríamos resolver cualquier eventualidad utilizando los otros medios de los que disponemos en la UNED. Este paso nos obligaba a tener en funcionamiento el site al comienzo del nuevo plazo de matrícula (julio). Pero lo que significaba para nosotros esta apertura, era un compromiso de relación directa, de mantenimiento de las posibles expectativas creadas y de «abrir una puerta a una casa, que siempre ha de estar lista para recibir visitas».

Los primeros diseños estaban acabados para el mes de mayo, y el 31 de julio se publicó en la web la versión definitiva del site. La primera experiencia relevante fue darnos cuenta de la viveza con que arrancó el espacio, las visitas se multiplicaban, los correos realizando consultas y las primeras inscripciones, llegaron incluso en Agosto (mes no lectivo en España). Es decir, se constató la existencia de un sector del alumnado que aunaba el uso de Internet con una estratégicamente avanzada preparación de la asignatura. Síntomas del perfil de un alumno con el 
que, desde el mismo momento, se estableció la vinculación. El alumno empezó a formar parte de un primer esbozo de la comunidad de la asignatura, actuando con ésta antes de la fecha propuesta por el calendario oficial.

Aunque ésta es una historia muy superficial de los primeros meses de trabajo, y aunque volveremos a ella en la segunda parte del artículo, no la cerraremos sin resaltar una cuestión que a todo el que se enfrente a un trabajo a través de Internet le puede frenar. Nos estamos refiriendo a los conocimientos técnicos necesarios. En nuestro caso, sin ser absolutamente profanos en la materia, ninguno de los miembros del equipo docente era un experto en informática, ni en la creación de sites. Nuestros conocimientos previos de Internet estaban más relacionados con la investigación de usuarios, contenidos y comunicación, que con el trabajo real de creación, alojamiento y funcionamiento de un site. Nuestro horizonte informático era en aquel momento lo que se denomina, eufemísticamente, «a nivel de usuario».

Otra cuestión importante derivada de la experiencia de esta fase inicial es que el trabajo en Internet nos ha permitido introducir algunos cambios, mejoras, contenidos requeridos por los alumnos y otros materiales, de forma flexible durante el curso. De la misma manera que no fue necesario tener todo cerrado a la hora de publicar la "Guía», tampoco lo ha sido cuando hemos ido desarrollando el proceso normal de trabajo a lo largo del curso. El desarrollo habitual de la asignatura escapaba así a la rigidez industrial que parecía característica de la enseñanza a distancia, con todo previamente planificado y prefijado, asimilándose más a un proceso conjunto.

\subsection{PROPUESTA DE INVESTIGACIÓN}

La propuesta inicial que trasladamos era una invitación a participar en un equipo de investigación conjunto. Alumnos y profesores se comprometían a realizar las tareas asignadas según el siguiente criterio: los alumnos realizarían el trabajo de campo de la investigación y los profesores diseñarían el proyecto y llevarían a cabo los análisis iniciales. Los resultados de la investigación serían publicados en un informe final elaborado por los profesores previamente comentado por los alumnos. Además de esto, a los alumnos se les proporcionaban los materiales didácticos necesarios para la realización de su trabajo. En concreto, los profesores diseñamos un proyecto de investigación ${ }^{4}$ y los materiales para la realización de las entrevistas; los alumnos, debían realizar dos entrevistas, y enviar las transcripciones al equipo docente.

\footnotetext{
${ }^{4}$ Para ver cualquiera de los materiales citados pueden visitar la URL http://www.uned.es/112013. El documento al que se refiere el proyecto es un texto íntegro que se puede descargar o imprimir en el site.
} 
Experiencia de creación de una Comunidad Educativa Virtual

Como resultado de las peticiones de participación de los alumnos en todas las fases del proyecto, incluidas las tareas de análisis y la elaboración del informe, el reparto final quedó de la siguiente forma:

- Los profesores elaboramos: el proyecto de investigación, la guía para la realización de las entrevistas, la guía para el análisis de las mismas, el preinforme y un informe final.

- Los alumnos llevaron a cabo las dos entrevistas y las enviaron transcritas al equipo docente para ser analizadas. En la siguiente fase, hicieron los análisis de sus propias entrevistas y un comentario con las aportaciones que creyeron necesarias al preinforme elaborado previamente por los profesores. Estos comentarios serán utilizados al elaborar el informe final.

Como es fácil ver, la iniciativa de los alumnos ha enriquecido en gran manera la experiencia.

\begin{tabular}{|c|c|c|c|c|c|c|c|c|}
\hline & Fase 1 & Fase 2 & & Fase 3 & Fase 4 & Fase 5 & Fase 6 & Fase 7 \\
\hline Alumnos & & Inscripción & 施 & $\begin{array}{l}\text { Realización } \\
\text { de } \\
\text { entrevistas. } \\
\text { Transcripción } \\
\text { y envío. }\end{array}$ & & $\begin{array}{c}\text { Análisis de } \\
\text { entrevistas y } \\
\text { comentarios } \\
\text { al } \\
\text { preinforme }\end{array}$ & $\begin{array}{c}\text { Discusión } \\
\text { del } \\
\text { preinforme }\end{array}$ & \\
\hline Profesores & $\begin{array}{c}\text { Diseño, } \\
\text { creación y } \\
\text { elaboración } \\
\text { del site y del } \\
\text { proyecto de } \\
\text { investigación }\end{array}$ & & 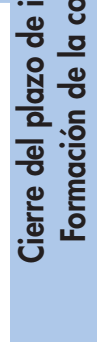 & & $\begin{array}{c}\text { Análisis } \\
\text { de las } \\
\text { entrevistas. } \\
\text { Elaboración } \\
\text { del } \\
\text { preinforme } \\
\text { y envío a } \\
\text { los alumnos }\end{array}$ & & $\begin{array}{c}\text { Discusión } \\
\text { del } \\
\text { preinforme }\end{array}$ & $\begin{array}{l}\text { Elaboración } \\
\text { del } \\
\text { informe } \\
\text { final }\end{array}$ \\
\hline
\end{tabular}

Figura 1. Programa de trabajo de la investigación. 


\subsection{ESTRUCTURA DEL SITE}

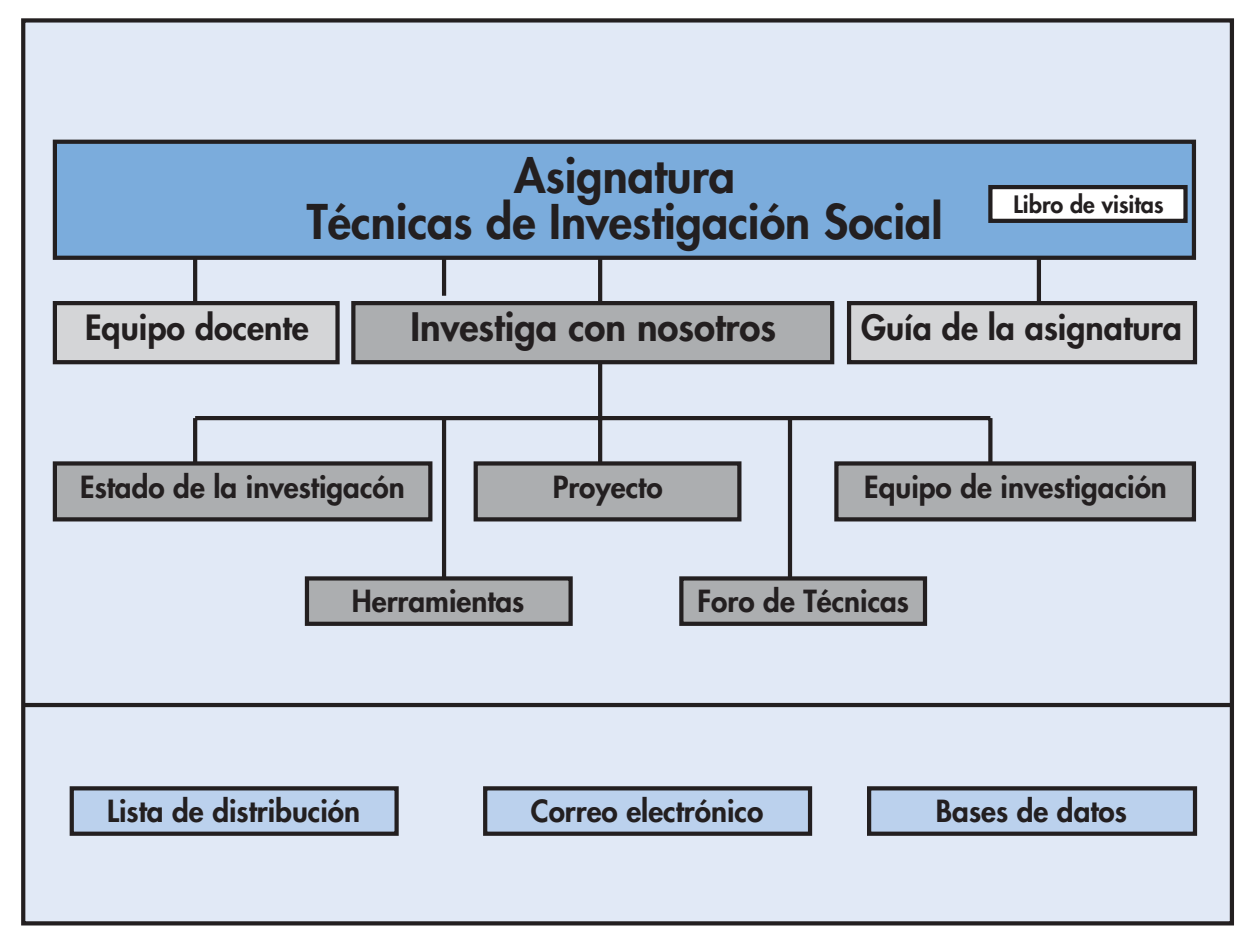

Figura 2. Estructura del site.

Como se puede observar en la figura 2, el site fue construido sobre tres grandes espacios de trabajo (dos online y uno offline). El primero, se reservó a la zona institucional. En él, se ofrece toda la información general. Páginas como "Guía de la asignatura», «Equipo docente», y otras que no aparecen en la figura 2, como «Exámenes», «Programa» y «Trabajos a realizar» constituyen este espacio. La segunda gran zona del site, la integran todas las páginas diseñadas para soportar online la experiencia práctica: «Investiga con nosotros», «Estado de la investigación», Proyecto», «Equipo de investigación», «Herramientas» y «Foro de técnicas» construyen este segundo espacio. Finalmente, la lista de distribución, las bases de datos, el correo-e y el software instalado para controlar el tráfico del site, constituyen las aplicaciones de trabajo offline.

Una vez que tuvimos claro los contenidos, nos preguntamos sobre la forma de estructurarlos: ¿quién y con qué objeto nos va a visitar? Teniendo presente que no podíamos abarcar con el diseño todas los objetivos que los alumnos se planteasen en su visita, lo hicimos para los dos tipos de visita más generales: el que solo busca información y el que participa en la investigación. 
2.3.1. Visita para el alumno que busca información general de la asignatura

La visita del alumno que tan solo quiere obtener información acerca de la asignatura sería fundamentalmente un paseo por la zona institucional. Quizá una vez conseguida la información buscada, éste tipo de alumno sólo volvería esporádicamente. Siguiendo este razonamiento, podríamos considerar que el problema fundamental de este visitante sería la localización de contenidos, por lo que la claridad en la navegación era el elemento más importante. Apoyándonos en estos criterios y en los trabajos que existen sobre los modos de navegación y la capacidad de encontrar contenidos en Internet según el diseño, (Krug 2001, Nielsen 1990) trazamos un recorrido que proporcionara accesos visibles desde la página principal a cada una de estas zonas. Los alumnos al entrar, se encuentran con una «barra de navegación»" (figura 3) que les ofrece a través de los siguientes enlaces la disyuntiva:

-institucional - área de investigación-:

\begin{tabular}{|c|c|c|c|}
\hline Eqaitso-docomits & Ging de in swargetura & 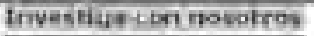 & Topotsatcat \\
\hline
\end{tabular}

Figura 3. Barra de navegación de la página principal.

Los dos enlaces de la izquierda (figura 3) proporcionan acceso a la zona institucional y los dos de la derecha dirigen la visita hacia las zonas de trabajo y comunicación del site. Una vez introducidos en la zona institucional, el diseño les propone una visita que recorre estas páginas con gráficos al efecto. Como pensamos que este alumno también tendría curiosidad por conocer el proyecto, le proporcionamos siempre la posibilidad de entrada desde cualquier página institucional a «investiga con nosotros» (entrada al área de investigación).

\subsubsection{El alumno que participa en la investigación}

La visita de este tipo de alumno se diseñó pensando en proporcionarle un espacio diferenciado de trabajo. Con este objeto, se crearon en esta zona páginas que ofrecían información exhaustiva de la investigación y de la situación en la que ésta y los materiales entregados, se encontraban. «Estado de la investigación» es la página en la que se describen cada una de las etapas por las que atraviesa el trabajo colectivo. A ella también se van incorporando informes del equipo docente y ejemplos de trabajos realizados por los alumnos. Los objetivos de la misma son: describir todas las fases por la que va pasando la investigación y ofrecer informes y documentos de interés para los alumnos. La otra página que está conectada con los objetivos de ésta, es «equipo de investigación». En ella, se

\footnotetext{
${ }^{5}$ La barra de navegación es un elemento que normalmente va en la parte superior de las páginas web y que proporciona enlaces a otras páginas de los sites.
} 
muestra a todos los alumnos los nombres y las direcciones de correo electrónico de los participantes en la investigación; sirviéndonos también como referencia para comprobar el estado en que se encuentran las entregas de trabajos.

Para dejar más claramente definido el espacio de trabajo, se le habilitó otra entrada alternativa (figuras 4 y 5). A través de ella, hicimos pública la invitación de participación y del proyecto. El formulario a través del cual los alumnos solicitaban su inclusión en la investigación también estaba situado en esta entrada. Esta página, «investiga con nosotros», ocultaba objetivos de seguridad. Con ella, pretendíamos crear un filtro para el foro y dar la seriedad suficiente como para que el formulario de alta no fuese utilizado por personas ajenas a la asignatura. En definitiva, estábamos trabajando en un espacio de acceso público. El marco institucional del site nos proporcionó un contexto lo suficientemente formal como para que el trabajo fuese respetado. Esto, que en principio creó bastantes dudas, ha funcionado bien por el momento.

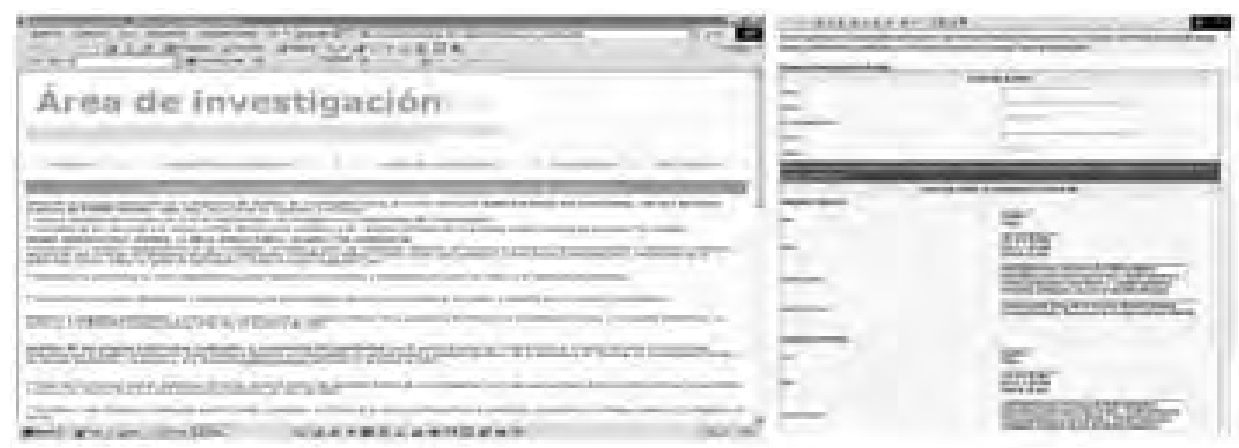

Figura 4. Página de entrada al área de investigación.

Figura 5. Detalle del formulario de envío.

La última página que nos queda por visitar es el «foro de técnicas». Espacio web de comunicación asincrónica ${ }^{6}$, permite a los alumnos publicar mensajes que son vistos por todo el grupo. Aloja también un tablón de anuncios que sirve al equipo docente para publicar convocatorias sobre cambios en el site o cualquier otra eventualidad. Sus objetivos eran servir de base de comunicación web a la comunidad educativa virtual.

Esta página ha tenido siempre un tratamiento especial para la navegación. Conscientes de que sería la página de visita diaria de los alumnos en muchas eta-

\footnotetext{
${ }^{6}$ Con comunicación asincrónica nos referimos a un tipo de comunicación en la que no existe un tiempo determinado entre la emisión del mensaje y la recepción del mismo por parte de otros participantes. El emisor escribe su mensaje, éste se publica es un espacio público y durante algún tiempo está disponible para los demás que pueden responderlo o simplemente leerlo.
} 
pas del proceso, la diseñamos accesible desde la páginas inicial del site y la pusimos en todas las barras de navegación.
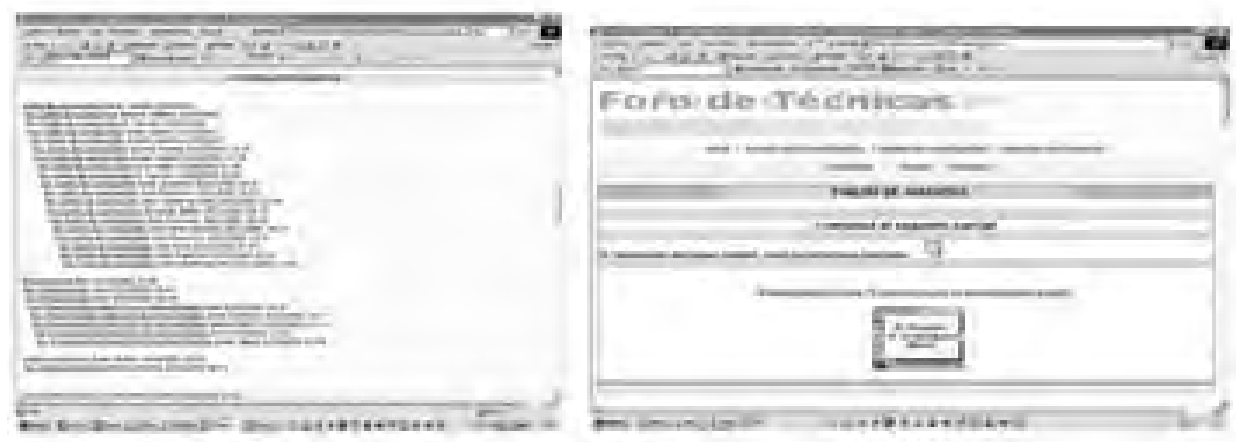

Figura 6. Foro de técnicas.

\section{SÍNTESIS DE RESULTADOS}

Antes de pasar al análisis, haremos una pequeña exposición de los resultados obtenidos hasta el momento.

Desde el punto de vista cuantitativo, la participación de los alumnos con respecto al total de matriculados, ha sido del $12 \%$. Esto significa que han participado en la experiencia un total de 90 alumnos.

La intervención activa de éstos en el foro ha rondado el 50\%. Hasta ahora han generado alrededor de 250 intervenciones divididas en 50 hilos de conversación. A través del correo electrónico han sido respondidas 30 consultas

Como materiales de investigación se han recogido 180 transcripciones de entrevistas.

El número de visitas realizadas al site (aunque éste es un dato con una fiabilidad discutible $)^{7}$ ronda las 4500 .

\footnotetext{
${ }^{7}$ Aunque los datos obtenidos se recogen por medio de software creado para este fin, sabemos que la fiabilidad de los mismos sólo puede ser tenida relativamente en cuenta. Aunque conocemos el número de entradas, alrededor de 4300 personas han entrado al site por la página principal (única forma de contabilizar acceso), nunca sabremos para qué entraron. Es decir, nos han visitado, pero ¿para qué? y ¿cuántas veces?, porque un mismo usuario puede entrar varias veces y contar. De todos modos tenemos más datos sobre la procedencia y podemos afirmar que la repetición sistemática de entradas sólo se daban desde los sitio en donde había alumnos participando en la investigación.
} 
Desde un punto de vista cualitativo, podríamos decir que los resultados más relevantes han sido los relacionados con la participación, las expectativas creadas y las iniciativas que los alumnos han aportado. El hecho de ser tomados en cuenta a la hora de construir la experiencia y el uso de nuevo métodos de acercamiento al profesorado, parece haber tenido un efecto claramente positivo en el alumnado ${ }^{8}$.

\section{REFLEXIÓN SOBRE LA EXPERIENCIA}

Una vez descrita la experiencia, volvamos la mirada hacia aquellos aspectos que pueden ser visiblemente mejorables en próximas ocasiones. De su revelación sólo cabe esperar una postura autocrítica, y como consecuencia de ésta, la intención de aportar experiencias que ayuden a salvar los errores por los que nosotros hemos pasado.

\subsection{SOBRE LAS CUESTIONES TÉCNICAS}

Aunque los problemas relacionados con el funcionamiento técnico del site nos preocupaban bastante al principio, finalmente se han mostrado como los más fáciles de solucionar. Aprender a construir páginas se convierte poco a poco en una cuestión de bricolaje, un trabajo rutinario en el que, con el tiempo, la construcción se subordina al diseño y el diseño se ajusta a los objetivos.

Planificar teniendo claros los objetivos docentes que queremos conseguir y pensar siempre en los medios y en los conocimientos heterogéneos de los que van a usarlo, fueron dos buenas referencias prácticas que sin duda ocuparon la parte más complicada de la tarea.

La organización de la inscripción de los alumnos, el correo devuelto, los problemas relacionados con la seguridad y la organización del foro, son las cuestiones técnicas que más problemas nos han generado.

Dar de alta a los alumnos ha resultado ser la tarea más tediosa de la investigación. La imposibilidad de poder unir una base de datos a un formulario que proporcionase al alumno un alta y a los profesores los datos necesarios, nos obligó a crear una fórmula compleja para comprobar y controlar las inscripciones. A través de un formulario en el que el alumno insertaba sus datos personales y la muestra que iba a entrevistar, éste solicitaba la inscripción. Cuando nosotros recibíamos estos datos, los pasábamos a dos bases, una de la muestra y otra de los participantes. Contra ese envío y una vez comprobado que los datos estaban co-

\footnotetext{
${ }^{8}$ Recomendamos visitar en el foro las presentaciones de los alumnos al grupo.
} 
rrectamente rellenados, se le remitía un correo con las guías para la realización de los trabajos y la comunicación del alta. Después de esto, se le inscribía en una lista de distribución y se le anotaba en la página web «equipo de investigación». Esta tarea burocrática se ha mostrado efectiva con el tiempo.

Sin embargo, todo este mecanismo se venía abajo cuando el alumno rellenaba incorrectamente sus datos personales o como ocurrió al principio, y debido a nuestra ignorancia, creamos un formulario en el que los campos no eran obligatorios. Este error aparentemente inocente, provocó bastantes altas defectuosas. Resolver las peticiones incompletas nos llevó dos semanas. Pero ¿por qué no rellenaban todo si estaba claro que había que hacerlo en las instrucciones? El problema de fondo es que normalmente quien no rellena bien un formulario, es porque no lo lee detenidamente (algo habitual en la web) o no tiene costumbre de hacerlo, y éste, normalmente tampoco tiene experiencia con el correo. En síntesis, lo que pasó fue que aquellos con menor experiencia rellenaban mal una inscripción que debían comprobar en un correo que no usaban. Con lo que hubo que llamarles por teléfono para explicarles el problema.

Solucionar este tema es complicado. Unir base de datos a la web, es una tarea que requiere de permisos del administrador y más conocimientos de los que nosotros teníamos. Por este motivo diseñamos algo muy manual pero que finalmente ha funcionado.

El correo es la mejor herramienta de comunicación que hemos utilizado. El mayor problema generado por éste, ha sido el de los correos devueltos. Cada envío general provoca alrededor de 5 ó 6 devoluciones por no encontrar al usuario. Esto se ha debido fundamentalmente a que muchos alumnos han usado servicios gratuitos de correo. Aparte de un funcionamiento más restringido, este tipo de servidores de correo requieren una visita semanal a los mismos; de lo contrario, el servidor elimina los mensajes. Si a este problema le añadimos que la falta de una visita al mes, supone la baja automática del usuario, nos encontraremos con lo que nos ha sucedido: un 10\% de alumnos han tenido que cambiar de dirección de correo durante la experiencia. El problema de gestión que esto supone se agrava con la pérdida de comunicaciones y materiales enviados. Hay varias vías para solucionar esto. En primer lugar, es necesario tener sitios alternativos en los que publicar materiales y convocatorias. En nuestro caso utilizamos el foro. En segundo lugar, el alumno debe tener claro los servicios que le ofrecen estos servidores y ha de mantener sus cuentas activas. Por último, la solución definitiva, que el propio servidor de la universidad les habilite una cuenta de correo a los participantes.

A pesar de haber sido escasos, los problemas de seguridad nos han hecho sentir «la fiebre del virus» en dos ocasiones. Si tenemos en cuenta el tráfico de documentos que ha habido entre los miembros del grupo y la obligación que teníamos de abrir estos documentos, entenderemos el aumento del riesgo con que 
han vivido nuestros PC. Los sistemas de detección de virus que la propia UNED posee han solucionado este problema. Aunque algún alumno ha remitido mensajes diciendo que se le habían enviado virus, hasta ahora siempre han sido falsas alarmas.

La última cuestión técnica digna de mencionar ha sido el funcionamiento del foro. La utilización de «Front-page 2000» como software para la construcción de sites, se podría valorar como positiva en casi todos los aspectos. La facilidad de uso, la claridad en las ayudas y la capacidad para gestionar las aplicaciones que lleva integradas, nos ha hecho muy fácil la realización de la experiencia. Si esta aplicación adolece de algo, es de la forma en que sus foros son construidos y gestionados. Lógicamente, la evolución de aplicaciones específicas para estas tareas ha avanzado a un ritmo que la versión utilizada por nosotros no ha podido incorporar. El control de usuarios, la organización de varios foros que convivan con el principal y la imposibilidad de adaptar el diseño de forma flexible al administrador, han sido los mayores problemas observados. Sin embargo, estamos seguros de que las nuevas versiones harán mejoras en este sentido. La relevancia que están teniendo las "comunidades virtuales», y las necesidades de una gestión efectiva de multitud de mensajes y el control de los participantes en ellas, así lo requiere. En la enseñanza a distancia es imprescindible una aplicación que gestione todo esta comunicación de mensajes y contenidos de manera efectiva.

\subsection{SOBRE LAS CUESTIONES SOCIALES}

Analizar los aspectos sociales surgidos durante la experiencia, es sin duda un ejercicio mediatizado por el ojo del observador y el contexto en el que éste mira e interviene (Alonso, 1998). En este caso, en el que el analista es además un «actor» implicado directamente en la acción, la subjetividad en los análisis toma especial relevancia. Ahora bien, una vez puesto esto de manifiesto, también debemos resaltar que la obtención de los datos utilizados para el análisis, ha sido llevada a cabo de una forma rigurosa.

Desde el principio teníamos claro que la experiencia podría servir también para entender la forma en que se cimienta lo social en estos espacios de comunicación electrónica. Percibíamos que muchas de las relaciones sociales que construyen la comunicación mediada por computadora (Licklider, 1968) no se modelan exclusivamente en la pantalla. Éstos planteamientos difieren de otras posiciones que, o bien proponen que la relación virtual ayuda a transformar y a ocultar la identidad del individuo en el medio (Turkle, 1997), o bien sugieren que la ausencia del cara a cara y la posibilidad de elección alternativa en este medio, provoca relaciones libres y simétricas entre los miembros participantes en una «comunidad» (Shapiro, 2001). Nuestra idea era que cuando en la relación virtual se parten de posiciones e identidades conocidas por los demás, como es el caso 
de las comunidades educativas virtuales, éstas reproducen en el espacio virtual las mismas relaciones sociales que en el espacio real.

La importancia de esta cuestión para la construcción de comunidades educativas virtuales en el futuro, era suficiente aliciente como para averiguar la forma en que esto se producía. A la vez que íbamos construyendo el site, se han recogido de forma sistemática todos los documentos y los asuntos más relevantes que han ido surgiendo de la experiencia. Podríamos decir, que para confeccionar estos resultados, al método etnográfico tradicional, se le ha unido una etnografía virtual (Hine, 2000) obtenida electrónicamente.

\subsubsection{Resultados del análisis}

Alumnos, técnicos y profesores son los tres grupos que han intervenido en el proceso. Sin embargo, todo lo sucedido no podría ser explicado sin tener en cuenta el contexto social proporcionado por la universidad (Augé, 1992).

La primera conclusión que podemos extraer del análisis nos indica que el orden social se ha reproducido en el espacio electrónico de forma sistemática. El comportamiento de los participantes en el medio electrónico (virtual) comparado con el comportamiento de éstos en el medio físico (real) no ha variado en exceso. Alumnos y profesores han mantenido los mismos modos de comportamiento. Si observamos las fórmulas de cortesía utilizadas en muchos correos electrónicos como elemento de interpretación, veremos que éstas no difieren en gran medida de las que se producen en el correo ordinario. Ejemplos como: «Estimado Sr. Profesor...», «Estimados alumnos» "...le saluda atentamente..» «Saludos...» y sobre todo el uso de un tratamiento formal y respetuoso, incluso en los momentos de conflicto, reafirman esta conclusión. No obstante, sí se puede observar algún cambio con respecto a esta relación cuando la comunicación se produce en el «foro de técnicas». A diferencia de la formalidad producida en el correo electrónico, el foro se ha entendido como un espacio más cotidiano y próximo de conversación. Siendo imposible en la experiencia la utilización del cara a cara en la conversación electrónica, esta forma de relación producida en el foro, es lo que más se asemeja a la conversación en la vida cotidiana (Goffman, 1981).

De esta manera, entendemos que tanto las presentaciones como las fórmulas empleadas para comunicarse, ofrecen claramente una identidad del alumno que pretende ser convertida en una autoría reconocible. Las presentaciones en el foro, abundan en datos personales que remarcan el sacrificio y la dificultad del estudio a distancia expresando también una valoración positiva ante la utilización de estas nuevas tecnologías. Más allá del valor de los contenidos de los mensajes emitidos, podemos observar que de una forma velada, la autoría de lo expresado también proporciona al alumno un acto de presentación prestigioso. 
¿Qué hubiera ocurrido si este espacio no hubiera sido público? Y aún más ¿qué hubieran dicho los alumnos si a este espacio no hubieran tenido acceso los profesores? A nuestro modo de ver, es evidente que las comunicaciones hubieran variado. De la misma forma que el correo-e se ha entendido como un acto de comunicación interpersonal y ha reproducido un modo de comunicación muy formal; el foro ha sido interpretado "como si» de un aula se tratara. En ese aula, la cotidianeidad proporciona relaciones de comunicación que eliminan algunas barreras levantadas por la interpretación de un rol asumido.

$\mathrm{Si}$ analizamos el origen de los conflictos surgidos con varios alumnos, veremos cómo interpretan éstos la relación. Después de varios meses de trabajo, aquel que manda un correo-e a los profesores, entiende que éstos deben conocer su nombre y sus circunstancias. De esta manera, se suceden los correos en los que los alumnos hablan de temas sin identificarse, continúan conversaciones a preguntas respondidas hace meses y, en definitiva, interpretan que su identidad es reconocida fácilmente. Si este re-conocimiento no se produce, surge el conflicto con virulencia. Parece como si el tiempo o el tamaño del grupo no fuesen coordenadas relevantes que explicaran correos en los que se pide una confirmación de autoría. Está claro que lo que se percibe es una relación directa y personal con el profesor.

Otra cuestión digna de significar ha sido el respeto a los planteamientos como zona de trabajo del foro. Si no ha existido ninguna intervención alejada de los objetivos marcados para el espacio y, éste era, además, un espacio de acceso público, podemos interpretar que al igual que otros espacios públicos se respetan en el medio físico, el medio virtual ofrece garantías parecidas.

Otro asunto que ha de ser puesto de manifiesto, es la relación de trabajo con los técnicos. Todo el proceso de construcción inicial se ha visto influenciado por las decisiones de este grupo. La inseguridad, la incapacidad de gestión del servidor y las dudas acerca de nuestros conocimientos, fueron excusas constantes para frenar algunas de las demandas. Las dificultades de encontrar un servidor en el que alojar con garantías el site, la poca ayuda recibida a la hora de enfrentarnos a la gestión técnica del mismo y las reticencias con las que este grupo nos ha tratado al no estar esta asignatura todavía contemplada como parte del proceso de virtualización institucional, hacen entrever una relación asimétrica que debe ser cambiada en el futuro. Ahora bien, también deja ver que es posible diseñar parte de la labor docente en la Red y de una manera que, por su relativamente escasa institucionalización, puede denominarse doméstica.

Era evidente, que en principio no poseíamos los conocimientos técnicos necesarios para interpretar todos los problemas que pueden surgir ante un proyecto como el que queríamos llevar a cabo; pero el tiempo y la ejecución del mismo, ha mostrado claramente que las dificultades técnicas no eran tan graves como para desecharlo. 
Experiencia de creación de una Comunidad Educativa Virtual

La construcción de espacios virtuales con objetivos docentes, ha de ser entendido por todos como un proyecto a realizar de manera conjunta. Los conocimientos de los técnicos son imprescindibles. Su colaboración es tan necesaria que los profesores sin su ayuda no se embarcarán en nada que no consideren posible. Ahora bien, la puesta en marcha de espacios, herramientas o plataformas sin contar con los conocimientos de los docentes, harán de éstos un "parto doloroso». Si hay algo claro es que toda la experiencia docente se ha de volcar en ellos. La peculiaridad de cada universidad exige espacios creados ad-hoc. Los productos «enlatados» pueden encontrarse con frenos y reticencias ante un uso difícil e incluso contrario a lo ya establecido. Por ello, es aún más imprescindible un proceso de colaboración en lugar de un proceso de imposición técnica. La influencia de lo social en este cambio de posiciones, sólo puede ser entendida desde la empatía. A veces la técnica no es capaz de soportar algunas iniciativas, pero los docentes podremos entender los motivos.

\section{CONCLUSIONES}

Mucho se ha escrito sobre la capacidad de transformación que Internet tendría en los sectores económicos, laborales, educativos o de comunicación (Castells 1997, Giddens 1999, Rifkin 2000, Broncano 2000, Negroponte 2000). Hemos vivido momentos en los que parecía que no necesitaríamos movernos de la pantalla para comprar, trabajar, relacionarnos, informarnos o estudiar. Tras el freno del marketing masivo, después de las dificultades del comercio electrónico, del hundimiento de muchas empresas «punto com», de las dudas con respecto al uso de los sistemas de información (Whitaker 1999) y, sobre todo, de la sensación de desfase entre expectativas creadas y lo que realmente se produce en la Red, debemos ser cautos con las perspectivas que plantean transformaciones profundas provocadas por Internet en la educación a distancia.

El acceso generalizado, el conocimiento del funcionamiento técnico del medio, el hábito en el uso o la capacidad para generar e interpretar estos nuevos modos de relación, están todavía lejos de convertirse en una práctica habitual del alumno según lo experimentado. No obstante, podemos concluir que la experiencia ha sido muy positiva y enriquecedora. La participación ha ido en todo momento más allá de nuestras mejores estimaciones. Si analizamos la repercusión del nuevo modelo de prácticas con los datos de cursos anteriores, comprobaremos que el incremento ha sido considerable (135\%). Por ejemplo, la realización del proyecto de investigación (trabajo del primer parcial) ha pasado de los 60-70 participantes de otros años, a los 95 de éste. Si a esto le añadimos los 70 alumnos que intervienen exclusivamente en la práctica (20 de los 90 participantes en el trabajo de Internet han realizado los dos ejercicios), nos encontramos con 165 alumnos que, de una forma u otra, realizan algún ejercicio práctico 
(aproximadamente el 23\% del total). Para las características del alumnado de la UNED, y tratándose de trabajos voluntarios, este porcentaje de participación se puede considerar como muy aceptable. Claro está, que el efecto de la calificación ha podido ayudar a incrementar la participación, pero si tomamos como referencia los testimonios de los alumnos, es indudable que la investigación ha supuesto un incentivo muy positivo para ellos.

La transformación producida en la vida de la asignatura por la dialéctica comunicativa entre profesores y alumnos, se deja ver en el interés de éstos últimos por transformar la experiencia en algo realmente propio. El descubrimiento de nuevas dinámicas de trabajo, la exposición de dificultades individuales en público o el conflicto creador ante la nueva situación comunicativa, nos conduce a poder declarar la potencialidad real del medio en un sentido interactivo. La relación comunicativa ha fomentado la comprensión de la materia de la asignatura. En algunos casos la discusión directa nos ha permitido eliminar los tabúes que existen alrededor de la complejidad de la misma. En suma, podríamos decir que la interacción ha sido una de las claves del éxito de la experiencia.

Por último, podríamos concluir que en gran parte hemos conseguido construir entre todos una comunidad educativa virtual. De lo sucedido, podemos deducir que los actores han convivido en un espacio interactivo de comunicación electrónico durante un curso, que se han incorporado de manera flexible nuevos materiales y que se han realizado de manera conjunta tareas docentes. Todo ello, nos conduce a declarar que la experiencia puede ser entendida como una muestra del potencial de la Red.

Cuando la comunicación, la relación social y la interacción se sitúan en el primer plano de la pantalla, Internet toma otra dimensión.

En síntesis, ésta podría ser la conclusión más importante de la experiencia. Internet nos permite acercarnos, pero depende de nosotros que eso sea factible. 
Experiencia de creación de una Comunidad Educativa Virtual

\section{REFERENCIAS BIBLIOGRÁFICAS}

Alonso, L. E. (1998). La mirada cualitativa en sociología. Una aproximación interpretativa, Madrid: Fundamentos.

Augé, M. (1992). Los no lugares. Espacios del anonimato. Una antropología de la sobremodernidad, Barcelona: Gedisa.

Broncano, F. (2000). Mundos artificiales. Filosofía del cambio tecnológico, México: Paidós.

Callejo, J. (2000). «La Demanda de Autonomía Tecnológica en la educación a distancia: una aproximación en el contexto de la UNED». Revista Iberoamericana de Educación a Distancia, Madrid: UNED. Vol. 3 ni 1, junio, pp. 27-50.

GoFFMAN, E. (1981). La presentación de la persona en la vida cotidiana, Buenos Aires: Amorrortu.

Hine, C. (2000). Virtual Ethnography, London: SAGE publications.

KRUG, S. (2001). No me hagas pensar, Madrid, Pearson Educación.

LÉvY, P. (1999). ¿Qué es lo virtual?, Barcelona: Paidós.

LickLIDER, J. (1960). «Man-Computer Symbiosis». IRE Transactions on Human Factors in Electronics. Vol. HFE-1, Marzo, pp. 4-11.

LickLIDER, J. (1968). «The computer as a Communication Device», Science and Technology. Abril.(Los dos artículos de Licklider, J. (1960 y 1968) están publicados en Internet en la dirección:http://gatekeeper.dec.com/pub/DEC/SRC/research-reports/SRC-061.pdf. Revisado y activo abril de 2002)

Negroponte, N. (2000). El mundo digital, Barcelona: Ediciones B.

Nielsen, J. (1999): Designing Web Usability: The Practice of Simplicity, Indianapolis: New Riders Publishing.

RHEINGOLD, H. (1994). La comunidad virtual. Una sociedad sin fronteras, Barcelona: Gedisa.

Rifkin, J. (2000). La era del acceso. La revolución de la nueva economía, Barcelona: Paidós.

SHAPIRo, A. (2002). El mundo en un clic. Cómo Internet pone el control en sus manos, Barcelona: Grijalbo Mondadori.

TuRKLE, S. (1997). La vida en la pantalla. La construcción de la identidad en la era de Internet, Barcelona: Paidós.

Whitaker, R. (1999). El fin de la privacidad. Cómo la vigilancia totales está convirtiendo en realidad, Barcelona: Paidós.

\section{PERFIL ACADÉMICO Y PROFESIONAL DEL AUTOR}

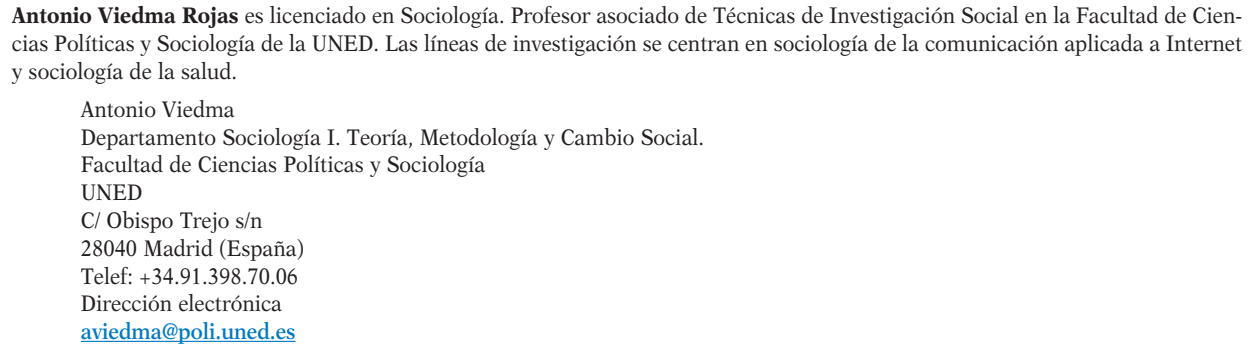

Antonio Viedma Rojas es licenciado en Sociología. Profesor asociado de Técnicas de Investigación Social en la Facultad de Ciencias Políticas y Sociología de la UNED. Las líneas de investigación se centran en sociología de la comunicación aplicada a Internet y sociología de la salud.

Antonio Viedma

Departamento Sociología I. Teoría, Metodología y Cambio Social.

Facultad de Ciencias Políticas y Sociología

UNED

C/ Obispo Trejo s/n

28040 Madrid (España)

Telef: +34.91.398.70.06

Dirección electrónica

aviedma@poli.uned.es 